\title{
A Predictive Route Maintenance Protocol Based on Signal Strength for Dense Ad Hoc Networks
}

\author{
Sharmila Sankar and V. Sankaranarayanan
}

\begin{abstract}
Ad hoc wireless networks consist of mobile network without base stations, and are characterized by a host in a highly dynamic network topology. The topology changes frequently due to host migration, signal interference and power outages, making the route maintenance a challenging consideration in designing routing protocols. Most of the existing protocols perform an end-to-end route discovery to establish a new connection for the communication during link breaks. Such route repair mechanism causes high control overhead and long packet delay in a dense network. In this paper, we propose an enhanced proactive route maintenance protocol, where a node that is likely to cause link error, as a preventive measure, hands off routing information to a new node by monitoring the signal strength of the received signals. The proposed protocol aims to reduce the probability of route breakage and hence reduces the control overhead and latency, and increases the packet delivery ratio. Through simulation, we show that the proposed protocol is simple, robust and effective.
\end{abstract}

Index Terms-Dense MANETs, RSSI, link stability, apriori maintenance.

\section{INTRODUCTION}

An ad hoc network is formed by a group of wireless devices without depending on any infrastructure. Each node communicates directly with its neighbors and functions as a router that forwards packets for nodes that are not within transmission range of the sender. Maintaining communication in ad hoc networks requires effective routing mechanisms in the presence of dynamic topology, which may cause route failures and requires discovery of new routes. Therefore, the routing protocol which aims at minimizing the control overhead should minimize the overhead from such maintenance.

Routing protocols for ad hoc networks can be categorized as proactive or reactive (on-demand) based on when routes are discovered. Proactive protocols maintain up-to-date routing information regardless of the presence of traffic, and so consume valuable resources such as bandwidth and power even if the network is idle. On-demand routing protocols have been shown to reduce routing overhead by only maintaining actively used routes.

Although on-demand routing protocols only initiate route discovery when a route is needed, such discovery is typically performed via network-wide flooding. Since flooding consumes a substantial amount of bandwidth, it is essential to reduce the frequency of route discoveries, and so network wide flooding. To overcome performance problems due to frequent route discovery attempts, hybrid protocols

Manuscript received June 1, 2012; revised July 2, 2012.

The authors are with the B S Abdur Rahman University, India (e-mail: sharmilasankar@yahoo.com). incorporate both reactive and proactive protocol characteristics. Although hybrid protocols do not waste resources by flooding the network for each route request, it is difficult to balance the cost of exchanging routing information periodically (i.e., proactivity) and network-wide flooding for route discovery (i.e., reactivity) [1].

Other protocols reduce the frequency of flooding by allowing a relay node to initiate a limited route discovery in the event of a route failure [2] or employ local error recovery mechanisms [3]. However, protocols using either limited broadcast or local error recovery have focused on reducing packet drops and not eliminating packet drops. Multipath routing protocols cache multiple routes to a destination in a single route discovery. However, in the presence of mobility, multipath protocols incur additional packet drops and delay due to their dependency on potentially stale routes from caches.

It is clear from the above discussions that, most of the protocols attempt to reduce packet drops, in order to increase the throughput and decrease the latency. Our goal is to design an efficient proactive local recovery protocol that initiates the route maintenance when a link break is expected rather than waiting for the break to occur.

\section{Motivation AND RELATED WORK}

In case of 802.11 ad-hoc networks, link management is done locally by each node for all nodes within its radio range. Also, this is done at the network layer by the routing protocol. The routing algorithms are thus equipped with mechanisms that aim to manage mobility, i.e. changes in the topology and routes. Both local and global approaches are used. Locally for link breakage detection, different mechanisms are employed, for example consecutive hello losses used in the DSR and the link expiration timers in AODV. Globally, it is done by periodically sending control packets to inform the entire network of topology changes and make it possible for nodes to recompute routes, or in a reactive way, sending a route error message to inform the source of route loss. Usually this does not involve using signal power measures obtained from lower layers since this information is unavailable. This leads to poor performance in terms of Packet Delivery Ratio (PDR) as it requires more time at this layer to manage links. In [4], [5], the authors discuss the various possibilities of using cross-layer feedbacks for the improvement of routing.

A set of signal based routing protocols have been proposed in [6-8]. They utilize the information available at the link level, to choose route. The signal quality of the channel, including signal strength, battery life, and location stability, are used to determine whether portions of the topology are stable or fluctuating, at any given time. The route 
establishment protocols proposed utilizes connectivity and link conditions to establish a route from the source to destination. However, message flooding issued by the source or an intermediate node is required to reestablish the route after the original route has been disconnected.

In this paper, we focus on local link management for efficiently managing mobility. For implementation we have used the Probabilistic Routing Protocol (PRP). Being a reactive protocol, it is well suited to dense ad hoc networks. With a cross layer approach it is able to anticipate breakages with the help of strength of the received signal. Moreover in a highly dense mobile network, there is a high probability of locating a new intermediate node in case of link errors. Hence instead of waiting for the link break to occur, a path can be repaired, once a weak link is identified by locating a new next hop neighbor. This leads to very less packet drops and reduces congestion caused due to flooding of route request in order to construct a new path on link breakage [9].

We first describe in brief PRP in next section. Then we propose our algorithms in section III and discuss its pros and cons. In section IV, we describe the simulations scenarios and discuss the results. Finally we conclude in section V, giving some future work directions.

\section{Probabilistic Routing Protocol Overview}

When a node wants to communicate with another node in the network a unique communication path is established between the sender and the receiver nodes. The source node scans the neighborhood vector for the destination. If the destination node is identified to be the single hop neighbor of the source, the source nodes starts transmitting data packets. The transmission of data will be uninterrupted until there is no change in the geographical positions of the source and the destination nodes.

The neighbors in the neighborhood vector are stored in the increasing order of their distances. The source node generates a RREQ packet and forwards it to $n / K$ neighbors (where $n$ is the total number of the neighbors and $\mathrm{K}$ - reachability parameter - a random number which depends on the network density) from the neighborhood vector targeting the farthest nodes from the source node [10], [11]. The intended neighbors check their neighborhood vectors and locate the destination else the same procedure is repeated till the destination is located.

The number of rebroadcasts is determined by the reachability parameter $\mathrm{K}$. The number of route requests to be rebroadcasted by each node to determine an optimal path depends on the chosen reachability parameter and the local density of the network. The reachability parameter $\mathrm{K}$ is directly proportional to the density D of the network. Due to higher connectivity, which is the inherent characteristics of a dense network, choosing even very few nodes to rebroadcast, discovers a path to the destination, which is closer to optimal path discovered by other broadcast protocols. The protocol is designed to work completely in a distributed manner and thus do not depend on any central entity.

\section{The Proposed Signal Strength Based Predictive ROUTE MAINTENANCE ALGORITHM}

Route failures have a significant negative impact on packet delivery. Packet dropping and higher delays are the main consequences of route failures. The time elapsed between link break detection and alternative path establishment can be high. Therefore, many studies have focused on improving route repair.

In PRP, link breaks are detected by lost acknowledgement packets or when a transmission is not overheard, during data transmission. It only detects the link breakage and do not repair it locally. The proposed algorithm attempts to fix the link errors at the earliest possible.

In outdoor environment, where wireless networks are usually deployed, the received power depends on the distance from which the packet was transmitted. The farther the transmitter is, the lower is the received power. This assumes we are in a free space (without obstacles). This measure is delivered by the wireless interface, and should be transmitted to the routing protocol. Thus a cross-layer approach is used.

This protocol uses signal strength as the measure of the degree of migration. A mobile host that move with a high speed at time $t$ is also expected to move with a high speed at time $t+1$, which makes the link to be unreliable initially and is likely to cause to cause link break in the future. The proposed protocol is named as Apriori Route Maintenance Protocol (ARMP).

\section{OPERATIONS OF ARMP}

This section first presents different cases when a route break can occur and then the details of ARMP protocol.

\section{A. Different Cases of Route Breaks}

Node $\mathrm{S}$ communicates with node $\mathrm{D}$ via the intermediate nodes A, B and C. Since all the nodes are mobile, any node may move in any direction at a given time. The four possible directions of movement and their impact are studied and discussed below as four different cases.

Case 1: An intermediate node a moving away from both its successor and predecessor node (Fig. 1) is considered as the first case. In this case the strength of both the received and the transmitted signal is sensed to be weak (i.e.) will fall below the threshold and hence the node A that sensed both the signals to be weak should proceed with finding a new intermediate node to handoff. The strength of the signal, after transmission, is found by overhearing.

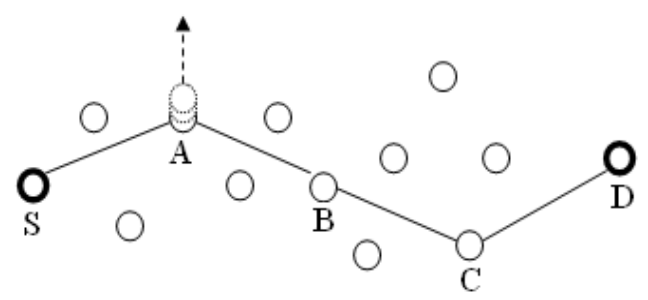

Fig. 1. Node a moving away from the predecessor and successor node.

Case 2: This is same as the previous case except for the direction of the movement and is handled in the same way (Fig. 2). 


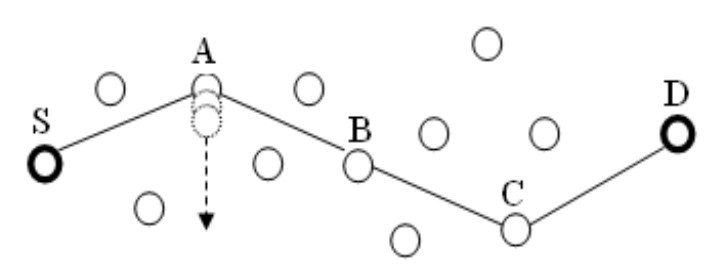

Fig. 2. Case 2.

Case 3: In this case, as shown in Fig. 3 the intermediate node $\mathrm{A}$ moves towards the node $\mathrm{B}$, thus making the link between itself and B stronger. But on the other hand the link between the node moving (i.e.) $\mathrm{A}$ and its predecessor becomes weak as the node A is moving away from its predecessor. Node A is likely to cause link break and hence as in the previous cases node A, may handoff by identifying a new common neighbor between its successor and predecessor. If no common neighbor is identified, A tries to identify a neighbor common to itself and its predecessor. If one such node is found, then the new node identified by a will act as the intermediate node between $\mathrm{A}$ and its predecessor, thus expanding the path length by one hop.

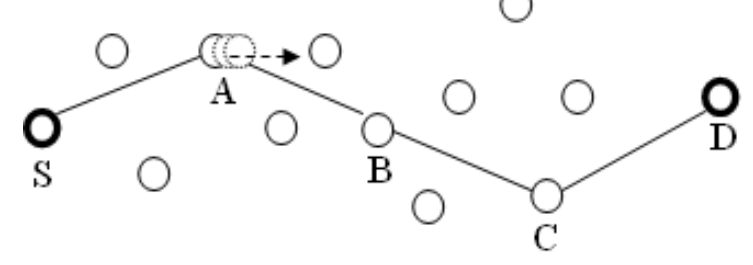

Fig. 3. Intermediate node a moving closer towards successor node B.

Case 4: Here the movement of the node a, make the link between itself and its successor weak. This case is same as that of the previous case but for the direction and it is handle in the same way as discussed in case 3 .

In all the above cases, it can be seen that, the link breaks are forecasted and the necessary action is taken by the node that is likely to cause the link break. This guarantees the improvement of the throughput, reduction of control overheads and packet loss.

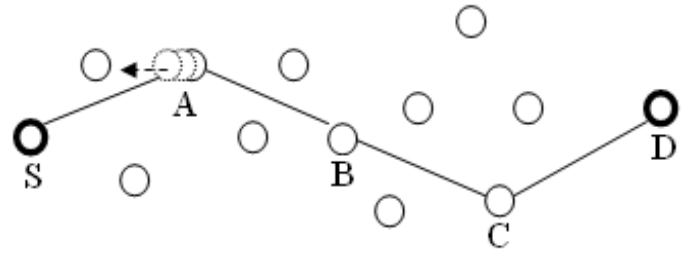

Fig. 4. Intermediate node a moving closer towards successor node $\mathrm{S}$

In all the above cases, it can be seen that, the link breaks are forecasted and the necessary action is taken by the node that is likely to cause the link break. This guarantees the improvement of the throughput, reduction of control overheads and packet loss.

\section{B. ARMP Protocol}

The proposed ARMP protocol utilizes the signal strength information, which is read from the Received Signal Strength Indicator (RSSI) value of the received packet. Indeed, received signal power level assessment is a necessary step in establishing a link for communication between wireless nodes. In ARMP protocol every node maintains a table that stores the connectivity information concerning each route (more than one route may pass through a host), including source host, destination host, predecessor, successor host, uplink and downlink stability, signal strength of the last packet received from the predecessor and overheard from the successor node in each route. This protocol maintains the route between the end hosts by avoiding link breaks in order to minimize the packet drops. At the first place, the node that is likely to cause the link break tries to handoff the routing path information to a new intermediate node. If no new intermediate between its successor and predecessor could be found, then it tries to expand the path by one hop by introducing a new intermediate node between itself and the predecessor/ the successor.

\section{1) Determination of handoff and path expansion}

Initially every node along the routing path records the strength of the signal received from its predecessor and successor node (by overhearing). On the reception of new packet, the signal received from the predecessor is compared with the recorded predecessor and successor values respectively. The sum of these two values is stored in the downlink and uplink stability fields of the routing table. In case of a node getting closer to the predecessor and moving away from the successor (Fig. 4), the downlink field will have negative value and uplink field will have a positive value stored in them. That is when a node moves away either from successor or from the predecessor node, the sum of the previously recorded signal strength and signal strength of the packet received currently will yield a positive value.

The route maintenance procedure is initiated by a node, when the sensed signal strength is less than $-90 \mathrm{dBm}$. The node that initiates the route maintenance proceeds to handoff by identifying a new neighbor node in common between its upstream and downstream node. If it is not successful, then the node tries to avoid the link break by expanding the routing path by one hop or two hops.

Algorithm:

\section{Procedure PathMaintenance}

$$
\{
$$

For each node in the network

\{

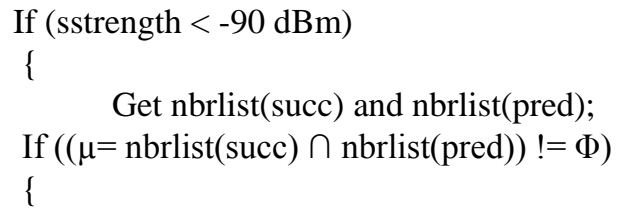

Select one of the nodes from $\mu$ as new intermediate node;

Modify the tables of new intermediate node, current node, and successor and predecessor node;

\}

Else

\{

Case1: uplink_stability $<0$ andand

downlink_stabilty $>0$

\{//Node moving towards successor Get nbrlist(pred); If $((\mu=\operatorname{nbrlist}($ curr $) \cap \operatorname{nbrlist}($ pred $)) !=\Phi)$ 
Select one of the nodes from $\mu$ as new intermediate node;

Modify the tables of new intermediate node, current node, and predecessor node;

\} // Path length increases by one hop \}

Case 2: downlink_stability $<0$ andand uplink_stability $>0$

\{//Node moving towards predecessor

Get nbrlist(succ); \{

If $((\mu=\operatorname{nbrlist}($ curr $) \cap \operatorname{nbrlist}($ succ $)) !=\Phi)$

Select one of the nodes from $\mu$ as new intermediate node;

Modify the tables of new intermediate node, current node, and successor node;

\} // Path length increases by one hop

\}

Case 3: uplink_stability andand downlink_stability > $0 / /$ Node moving away from successor and predecessor

Get nbrlist(succ) and nbrlist(pred); If $((\mu 1=\operatorname{nbrlist}($ succ $) \cap \operatorname{nbrlist}($ curr $)) !=\Phi)$ \{ If $((\mu 2=\operatorname{nbrlist}($ curr $) \cap \operatorname{nbrlist}($ pred $)) !=\Phi)$

Select a node from $\mu 1$ and $\mu 2$ as new intermediate nodes;

Modify the tables of new intermediate nodes, current node, successor and predecessor node;

\} // Path length increases by two

\}

\}

Default: Generate a warning message and send it to the upstream node;

\} // end else

\} // end if

\} // end for

\} //end procedure

\section{Simulation Results}

Normalized routing overhead is the number of routing packets transmitted per data packet which is delivered at the destination. Fig. 5 and Fig. 6 shows the routing overhead generated throughout the entire simulations with varying the number of connections (traffic flows). Most of routing packets are generated in the route discovery process. Route request messages are forwarded to the destination by broadcast in AODV and using border node retransmission technique in PRP-ARMP. The overall control overhead savings is very obvious at high density, compared with AODV. Thus PRP-ARM helps in reduction of congestion level caused by control packet and in better bandwidth utilization.

\section{Analysis}

Overhead gives an insight of the network bandwidth consumed by routing packets with respect to connection request. Fairly stable overhead is desirable property when considering the performance as it would indicate that overhead remains linear with varying parameters. From Fig.
5 and Fig. 6 it is observed that the overhead generated in PRP-ARMP is almost linear with varying densities whereas the overhead generated increases with the increase in the network density. With the increase in number of source nodes, an increase in the routing overhead can be observed from Fig. 5 and Fig. 6, but it is almost linear unlike AODV. From Fig. 7 it can be seen that initially there is increase in the routing overhead with increase in the node density but the routing overhead decreases in higher node densities. At low density, PRP-ARMP does not show much advantage. Especially when node number equals to 50 , the connectivity of the whole network is not quite good and partitioning is severe. Most of the transmission is successful only in small partitions with short route length. In such situation, the local recovery covers most portion of the whole partition already, thus we cannot see obvious control packet saving at low density. Increase in routing overhead is less with increase in number of source node, also the highest value for total routing packets is around 60 in case of AODV whereas 600 in case of PRP-ARMP. The new proposed protocol PRP-ARMP uses border node rebroadcasting for route discovery and enables the local repair of routes (bridging) thus drastically reducing the number of overhead packets as can be verified from Fig. 7.

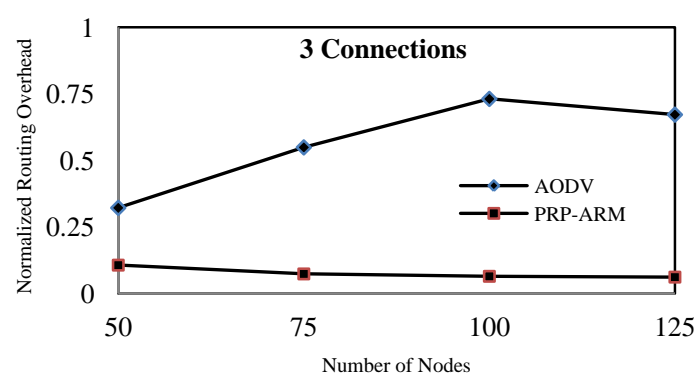

Fig. 5. Control overhead for 3 connections.

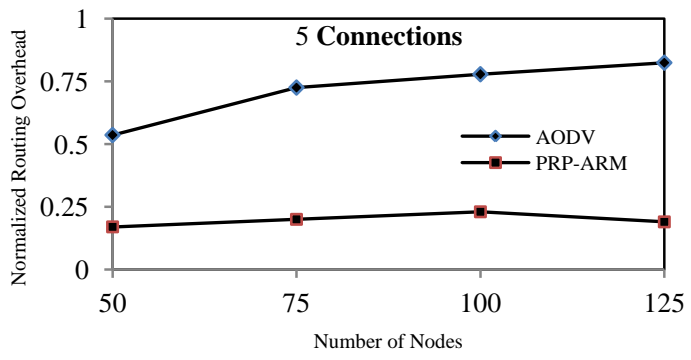

Fig. 6. Control overhead for 5 connections.

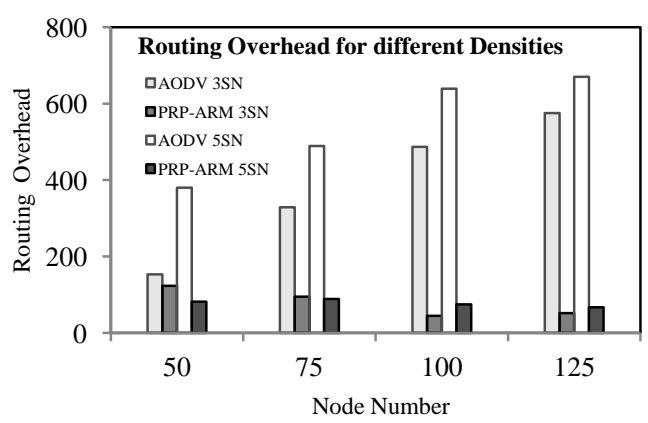

Fig. 7. Routing overhead (RERR+ RREQ+RREP) for AODV and PRP-ARM.

Local Recovery Success Ratio: The ratio of successful local recovery for various densities is collected. With low densities, where the network is sparse, the success ratio of 
PRP-ARMP is very low (Fig. 8). However, as the density goes higher, the connectivity of the network becomes higher; transmission with longer route length can be formed. In such a situation, the local recovery starts to show obvious advantage over end-to-end recover scheme, as the local recovery only requests its upstream and downstream neighbor, while the end-to-end recovery used in other reactive protocols like AODV and DSR floods the entire network. Successful local recovery ratio goes higher as the average degree of node goes higher.

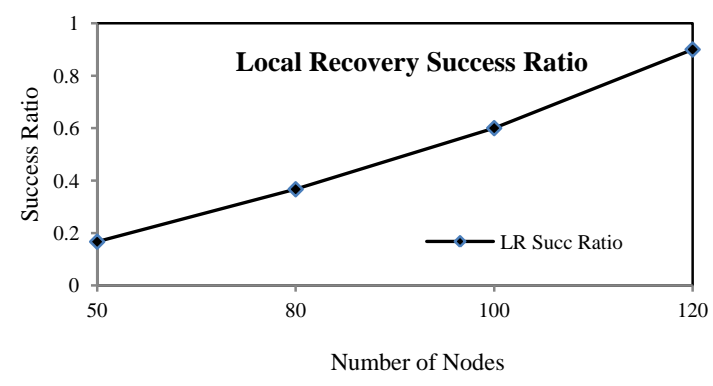

Fig. 8. Successful local recovery ratio.

\section{CONCLUSION}

In this paper, we presented route maintenance mechanism, called Apriori Route Maintenance, for dense mobile ad-hoc networks, that initiates proactive path switches when the quality of a path in use becomes suspect. An attempt has been made to improve the data connectivity in the network by selecting an alternate path as soon as the signal strength falls below the threshold value. In contrast to the end-to-end recovery mechanism, ARMP relies on strength of the received signal and nodal density within the region where breakage is likely to occur. As ARMP only disturbs the adjacent nodes, i.e. upstream and downstream nodes, it will mostly outperform end-to-end recovery in scenarios with big map size and high density, in which it only relies on a small percentage of the whole network to achieve the route maintenance process. Hence, it is a scalable solution for the route maintenance process. We showed that this method avoids using a path that is about to fail and eliminates the associated costs of detecting the failure and recovering from it, significantly improving the performance of the dense mobile ad hoc network.

\section{REFERENCES}

[1] Ramasubramanian, Z. J. Haas, and E. G. Sire, "SHARP: A hybrid adaptive routing protocol for mobile ad hoc networks," in Proceedings of 4th ACM International Symposium on Mobile Ad Hoc Networking and Computing (MobiHoc), 2003.

[2] C. E. Perkins, E. M. Royer, and S. R. Das, Ad hoc on-demand distance vector (AODV) routing, IETF RFC 3561, June 2003.

[3] M. Spohn and J. J. Garcia-Luna-Aceves, "Neighborhood aware source routing," in Proceedings of 2nd ACM International Symposium on Mobile Ad Hoc Networking and Computing (MobiHoc), 2001.

[4] K. Saravanan and T. Ravichandran, "A Cross layer based high throughput MAC protocol for 802.11 multi-hop as hoc networks," Eurosip Journal of Scientific Research, vol. 33, no. 4, 2009.

[5] E. Weiss et al., Improving routing performance in wireless ad hoc networks using cross-layer interactions in: Ad hoc Networks, vol. 5, issue 5, July 2007

[6] U. Jayahkudy, C. E. Tan, and T. Roth, "Link Signal Strength based Path Selection Scheme for Ad hoc Networks," IEEE conference Internet Technology and Secured Transactions, 2010

[7] Y. Taj and K. Faez, "Signal Strength Based Reliability: A Novel Routing Metric in MANETs," in Proceedings of Second International Conference on Networks Security, Wireless Communications and Trusted Computing, 2010.

[8] H. Ahn and S.-B. Rhee, "Simulation of a RSSI-Based Indoor Localization System Using Wireless Sensor Network," in Proceedings of the 5th International Conference on Ubiquitous Information Technologies and Applications (CUTE), 2010

[9] C. Gul and P. Mohapatra, "A framework for self-healing and optimizing routing techniques for mobile ad hoc networks," Wireless Networks, Springer-Verlag, 2008.

[10] S. Sankar and V. Sankaranarayanan, "A low overhaed reachability guaranteed dynamic route discovery mechanism for dense MANETs," International Journal of Ad hoc, Sensor and Ubiquitous Computing (IJASUC) vol.1, no.3, September 2010

[11] S. Sankar and V. Sankaranarayanan, "Framework for Probabilistic Routing Protocol in Dense MANETs," Recent Trends in Networks and Communications in Computer and Information Science, vol. 90, Part 2, pp. 447-456, 2010. 